УДК 517.5

B. V. Vynnyts'Kyi, R. V. Khats'

\title{
COMPLETE BIORTHOGONAL SYSTEMS OF BESSEL FUNCTIONS
}

\author{
B. V. Vynnyts'kyi, R. V. Khats'. Complete biorthogonal systems of Bessel functions, Mat. Stud. \\ 48 (2017), 150-155. \\ Let $\nu \geq-1 / 2$ and $\left(\rho_{k}\right)_{k \in \mathbb{N}}$ be a sequence of nonzero complex numbers such that $\rho_{k}^{2} \neq \rho_{m}^{2}$ \\ for $k \neq m$. We prove that if the system $\left\{\sqrt{x \rho_{k}} J_{\nu}\left(x \rho_{k}\right): k \in \mathbb{N}\right\}$ of Bessel functions of the first \\ kind of index $\nu \geq-1 / 2$ is exact (i.e. complete and minimal) in the space $L^{2}(0 ; 1)$, then its \\ biorthogonal system is also exact in $L^{2}(0 ; 1)$.
}

1. Introduction and main result. A system of elements $\left\{u_{n}: n \in \mathbb{N}\right\}$ in a separable Hilbert space $H$ is said to be exact $([14,15])$ if it is both complete (i.e. $\overline{\operatorname{span}}\left\{u_{n}: n \in \mathbb{N}\right\}=H$ ) and minimal (i.e. $u_{n_{0}} \notin \overline{\operatorname{span}}\left\{u_{n}: n \in \mathbb{N} \backslash\left\{n_{0}\right\}\right\}$ for each $n_{0} \in \mathbb{N}$ ). For every exact system $\left\{u_{n}: n \in \mathbb{N}\right\}$ there exists ([14, p. 28], [15]) a unique biorthogonal system $\left\{v_{m}: m \in \mathbb{N}\right\}$ such that $\left\langle u_{n} ; v_{m}\right\rangle=\delta_{m n}$, where $\langle\cdot ; \cdot\rangle$ is the inner product in $H$ and $\delta_{m n}$ is the Kronecker delta.

Let $\left(\rho_{k}\right)_{k \in \mathbb{N}}$ be an arbitrary sequence of distinct nonzero complex numbers. R. Young [15] proved that if the system of complex exponentials $\left\{e^{i \rho_{k} x}: k \in \mathbb{N}\right\}$ is exact in $L^{2}(-\pi ; \pi)$, then its biorthogonal system is also exact in $L^{2}(-\pi ; \pi)$.

Let

$$
J_{\nu}(z)=\sum_{k=0}^{\infty} \frac{(-1)^{k}(z / 2)^{\nu+2 k}}{k ! \Gamma(\nu+k+1)}, \quad z=x+i y=r e^{i \theta}
$$

be the Bessel function of the first kind of index $\nu \in \mathbb{R}$. Various approximation properties of the system

$$
\left\{\sqrt{x \rho_{k}} J_{\nu}\left(x \rho_{k}\right): k \in \mathbb{N}\right\}
$$

were investigated in a number of papers (see, for instance, $[2,5],[7]-[13]$ ). In particular, in $[9,10]$ it is found criteria for the completeness, minimality and basicity of the system (1) in $L^{2}(0 ; 1)$ if $\nu \geq-1 / 2$. Those results are formulated in terms of sequences of zeros of functions from certain classes of entire functions. The purpose of this paper is to prove the following theorem.

Theorem 1. Let $\nu \geq-1 / 2$ and $\left(\rho_{k}\right)_{k \in \mathbb{N}}$ be a sequence of nonzero complex numbers such that $\rho_{k}^{2} \neq \rho_{m}^{2}$ for $k \neq m$. If the system (1) is exact in $L^{2}(0 ; 1)$, then its biorthogonal system is also exact in $L^{2}(0 ; 1)$.

2010 Mathematics Subject Classification: 30B60, 33C10, 42A65, 42C30, 30D15, 30D20, 44A20, $46 \mathrm{E} 30$.

Keywords: Bessel function; entire function of exponential type; complete system; minimal system; biorthogonal system; exact system; orthonormal basis.

doi:10.15330/ms.48.2.150-155

(C) B. V. Vynnyts'kyi, R. V. Khats', 2017 
2. Preliminaries. Let $\nu \geq-1 / 2$ and $P W^{2, \nu}$ be the space of all entire functions $f$ of exponential type $\sigma \leq 1$ satisfying

$$
\|f\|_{P W^{2, \nu}}^{2}:=\int_{-\infty}^{+\infty}|x|^{2 \nu+1}|f(x)|^{2} d x<+\infty
$$

with the inner product

$$
\left\langle f_{1} ; f_{2}\right\rangle_{P W^{2, \nu}}=\int_{-\infty}^{+\infty}|x|^{2 \nu+1} f_{1}(x) \overline{f_{2}(x)} d x
$$

Lemma 1 ([3]). Let $\nu>-1$. Then every function $f \in L^{2}(0 ;+\infty)$ can be represented in the form

$$
f(z)=\int_{0}^{+\infty} \sqrt{z t} J_{\nu}(z t) h(t) d t
$$

with some function $h \in L^{2}(0 ;+\infty)$. In this case, $\|f\|_{L^{2}(0 ;+\infty)}=\|h\|_{L^{2}(0 ;+\infty)}$ and

$$
h(t)=\int_{0}^{+\infty} \sqrt{z t} J_{\nu}(z t) f(z) d z .
$$

Lemma 2 ([1, 4]). Let $\nu \geq-1 / 2$. A function $f$ has the representation

$$
f(z)=\int_{0}^{1} \sqrt{z t} J_{\nu}(z t) h(t) d t
$$

with some function $h \in L^{2}(0 ; 1)$ if and only if $f \in L^{2}(0 ;+\infty)$ and $f(z)=z^{\nu+1 / 2} Q(z)$, where $Q$ is an even entire function of exponential type $\sigma \leq 1$.

To prove our main result we need the following auxiliary statements.

Lemma $3([9,10])$. Let $\nu \geq-1 / 2$ and $\left(\rho_{k}\right)_{k \in \mathbb{N}}$ be a sequence of nonzero complex numbers such that $\rho_{k}^{2} \neq \rho_{m}^{2}$ for $k \neq m$. For a system (1) to be incomplete in $L^{2}(0 ; 1)$ it is necessary and sufficient that a sequence $\left(\rho_{k}\right)_{k \in \mathbb{N}}$ is a subsequence of zeros of some nonzero even entire function $G \in P W^{2, \nu}$.

Lemma $4([9,10])$. Let $\nu \geq-1 / 2$ and $\left(\rho_{k}\right)_{k \in \mathbb{N}}$ be a sequence of nonzero complex numbers such that $\rho_{k}^{2} \neq \rho_{m}^{2}$ for $k \neq m$. A necessary and sufficient condition for a system (1) to be complete and minimal in $L^{2}(0 ; 1)$ is that the sequence $\left(\rho_{k}\right)_{k \in \mathbb{Z} \backslash\{0\}}$, where $\rho_{-k}:=-\rho_{k}, k \in \mathbb{N}$, be a sequence of zeros of some even entire function $G \notin P W^{2, \nu}$ such that

$$
\left(z^{2}-\rho_{1}^{2}\right)^{-1} G(z) \in P W^{2, \nu}
$$

Moreover, the functions from biorthogonal system $\left\{\gamma_{k}: k \in \mathbb{N}\right\}$ are defined by equality

$$
\overline{\gamma_{k}(t)}=\frac{2}{\rho_{k}^{\nu-1 / 2} G^{\prime}\left(\rho_{k}\right)} \int_{0}^{+\infty} \frac{\sqrt{z t} J_{\nu}(z t) z^{\nu+1 / 2} G(z)}{z^{2}-\rho_{k}^{2}} d z, \quad t \in(0,1) .
$$

Besides, we have

$$
\int_{0}^{1} \sqrt{t z} J_{\nu}(t z) \overline{\gamma_{n}(t)} d t=\frac{2 z^{\nu+1 / 2} G(z)}{\rho_{n}^{\nu-1 / 2} G^{\prime}\left(\rho_{n}\right)\left(z^{2}-\rho_{n}^{2}\right)}, \quad n \in \mathbb{N},
$$

and

$$
\left\|\gamma_{k}(t)\right\|_{L^{2}(0 ; 1)}=\left\|\frac{2 G(z)}{\rho_{k}^{\nu-1 / 2} G^{\prime}\left(\rho_{k}\right)\left(z^{2}-\rho_{k}^{2}\right)}\right\|_{P W^{2, \nu}} .
$$


Remark 1. Let $P_{\nu}(z)=z^{-\nu} J_{\nu}(z)$ and $\left(\widetilde{\rho}_{k}\right)_{k \in \mathbb{N}}$ is a sequence of positive zeros of $J_{\nu}$. Then, by Lemma 4 , the system $\left\{\gamma_{k}\left(t ; P_{\nu}\right): k \in \mathbb{N}\right\}$, where

$$
\overline{\gamma_{k}\left(t ; P_{\nu}\right)}:=\frac{2}{\widetilde{\rho}_{k}^{\nu-1 / 2} P_{\nu}^{\prime}\left(\widetilde{\rho}_{k}\right)} \int_{0}^{+\infty} \frac{\sqrt{z t} J_{\nu}(z t) z^{\nu+1 / 2} P_{\nu}(z)}{z^{2}-\widetilde{\rho}_{k}^{2}} d z,
$$

is a biorthogonal system for the system $\left\{\sqrt{t \widetilde{\rho}_{k}} J_{\nu}\left(t \widetilde{\rho}_{k}\right): k \in \mathbb{N}\right\}$. Besides, we have

$$
J(k ; \nu):=\left\|\sqrt{t \widetilde{\rho}_{k}} J_{\nu}\left(t \widetilde{\rho}_{k}\right)\right\|_{L^{2}(0 ; 1)}=\left(\frac{\widetilde{\rho}_{k}}{2} J_{\nu+1}^{2}\left(\widetilde{\rho}_{k}\right)\right)^{1 / 2},
$$

the system

$$
\left\{\frac{\sqrt{t \widetilde{\rho}_{k}} J_{\nu}\left(t \widetilde{\rho}_{k}\right)}{J(k ; \nu)}: k \in \mathbb{N}\right\}
$$

forms an orthonormal basis in $L^{2}(0 ; 1)$ (see $\left.[5,7,8,13]\right)$ and thus each function $q \in L^{2}(0 ; 1)$ expanded into a convergent in $L^{2}(0 ; 1)$ series:

$$
q(t)=\sum_{k \in \mathbb{N}} a_{k} \frac{\sqrt{t \widetilde{\rho}_{k}} J_{\nu}\left(t \widetilde{\rho}_{k}\right)}{J(k ; \nu)} .
$$

In this case, (here and so on by $c_{j}$ we denote positive constants) $1 / c_{1} \leq J(k ; \nu) \leq c_{1}$ ([7]),

$$
\begin{gathered}
a_{k}=\int_{0}^{1} q(t) \frac{\sqrt{t \widetilde{\rho}_{k}} J_{\nu}\left(t \widetilde{\rho}_{k}\right)}{J(k ; \nu)} d t=J(k ; \nu) \int_{0}^{1} q(t) \overline{\gamma_{k}\left(t ; P_{\nu}\right)} d t, \quad \sum_{k \in \mathbb{N}}\left|a_{k}\right|^{2}<+\infty, \\
\int_{0}^{1} \sqrt{t z} J_{\nu}(t z) \overline{\gamma_{k}\left(t ; P_{\nu}\right)} d t=\frac{2 z^{\nu+1 / 2} P_{\nu}(z)}{\widetilde{\rho}_{k}^{\nu-1 / 2} P_{\nu}^{\prime}\left(\widetilde{\rho}_{k}\right)\left(z^{2}-\widetilde{\rho}_{k}^{2}\right)},
\end{gathered}
$$

and

$$
\left\|\frac{\sqrt{t \widetilde{\rho}_{k}} J_{\nu}\left(t \widetilde{\rho}_{k}\right)}{J(k ; \nu)}\right\|_{L^{2}(0 ; 1)}=J(k ; \nu)\left\|\overline{\gamma_{k}\left(t ; P_{\nu}\right)}\right\|_{L^{2}(0 ; 1)}=\left\|\frac{2 J(k ; \nu) P_{\nu}(z)}{\widetilde{\rho}_{k}^{\nu-1 / 2} P_{\nu}^{\prime}\left(\widetilde{\rho}_{k}\right)\left(z^{2}-\widetilde{\rho}_{k}^{2}\right)}\right\|_{P W^{2, \nu}}=1 .
$$

Lemma 5. Let $\nu \geq-1 / 2$ and $\left(\rho_{k}\right)_{k \in \mathbb{N}}$ be a sequence of nonzero complex numbers such that $\rho_{k}^{2} \neq \rho_{m}^{2}$ for $k \neq m$. If the sequence $\left(\rho_{k}\right)_{k \in \mathbb{Z} \backslash\{0\}}$, where $\rho_{-k}:=-\rho_{k}, k \in \mathbb{N}$, is a sequence of zeros of some even entire function $G$ satisfying $\left(z^{2}-\rho_{1}^{2}\right)^{-1} G(z) \in P W^{2, \nu}$, then for any $z=x+i y=r e^{i \theta}$ holds

$$
\left|z^{\nu+1 / 2} G(z)\right| \leq c_{2} e^{|\operatorname{Im} z|}(1+|\operatorname{Im} z|)^{-1 / 2}\left(1+|z|^{2}\right) .
$$

Proof. Since $([5,13])$

$$
\left|\sqrt{z} J_{\nu}(z)\right| \leq c_{3} e^{|\operatorname{Im} z|}\left(\frac{|z|}{1+|z|}\right)^{\nu+1 / 2}, \quad z \in \mathbb{C},
$$

by Lemmas 1 and 2, for all $z=x+i y=r e^{i \theta}$ we get

$$
\begin{gathered}
\left|z^{\nu+1 / 2}\left(z^{2}-\rho_{1}^{2}\right)^{-1} G(z)\right|=\left|\int_{0}^{1} \sqrt{z t} J_{\nu}(z t) h(t) d t\right| \leq \\
\leq\left(\int_{0}^{1}|h(t)|^{2} d t\right)^{1 / 2}\left(\int_{0}^{1}\left|\sqrt{z t} J_{\nu}(z t)\right|^{2} d t\right)^{1 / 2} \leq c_{4}\|h\|_{L^{2}(0 ; 1)} e^{|\operatorname{Im} z|}(1+|\operatorname{Im} z|)^{-1 / 2} .
\end{gathered}
$$

This yields the required estimate. Lemma 5 is proved. 
3. Proof of Theorem 1. Suppose that the biorthogonal system $\left\{\gamma_{k}(t): k \in \mathbb{N}\right\}$ is not complete in $L^{2}(0 ; 1)$ and that, for example, $\rho_{1} \neq \widetilde{\rho}_{k}$ for all $k \in \mathbb{N}$. Then, according to Hahn-Banach theorem ([6, p. 131]), there exists a nonzero function $q \in L^{2}(0 ; 1)$ such that

$$
\int_{0}^{1} q(t) \overline{\gamma_{n}(t)} d t=0
$$

for all $n \in \mathbb{N}$. In view of Remark 1 , we obtain

$$
\begin{gathered}
0=\int_{0}^{1} q(t) \overline{\gamma_{n}(t)} d t=\sum_{k \in \mathbb{N}} \frac{a_{k}}{J(k ; \nu)} \int_{0}^{1} \sqrt{t \widetilde{\rho}_{k}} J_{\nu}\left(t \widetilde{\rho}_{k}\right) \overline{\gamma_{n}(t)} d t= \\
=\frac{1}{\rho_{n}^{\nu-1 / 2} G^{\prime}\left(\rho_{n}\right)} \sum_{k \in \mathbb{N}} \frac{a_{k}}{J(k ; \nu)} \frac{2 \widetilde{\rho}_{k}^{\nu+1 / 2} G\left(\widetilde{\rho}_{k}\right)}{\widetilde{\rho}_{k}^{2}-\rho_{n}^{2}}, \quad n \in \mathbb{N},
\end{gathered}
$$

whence

$$
\sum_{k \in \mathbb{N}} \frac{a_{k}}{J(k ; \nu)} \frac{2 \widetilde{\rho}_{k}^{\nu+1 / 2} G\left(\widetilde{\rho}_{k}\right)}{\widetilde{\rho}_{k}^{2}-\rho_{n}^{2}}=0, \quad n \in \mathbb{N}
$$

In the case $\widetilde{\rho}_{k}^{2}=\rho_{n}^{2}$ we assume that

$$
\frac{2 \widetilde{\rho}_{k} G\left(\widetilde{\rho}_{k}\right)}{G^{\prime}\left(\rho_{n}\right)\left(\widetilde{\rho}_{k}^{2}-\rho_{n}^{2}\right)}=1
$$

Let

$$
\widetilde{P}(z)=\sum_{k \in \mathbb{N}} \frac{2 a_{k} \widetilde{\rho}_{k}^{\nu+1 / 2} G\left(\widetilde{\rho}_{k}\right)}{J(k ; \nu)} \frac{P_{\nu}(z)}{\widetilde{\rho}_{k}^{2}-z^{2}} .
$$

Then

$$
\widetilde{P}\left(\rho_{n}\right)=P_{\nu}\left(\rho_{n}\right) \sum_{k \in \mathbb{N}} \frac{2 a_{k} \widetilde{\rho}_{k}^{\nu+1 / 2} G\left(\widetilde{\rho}_{k}\right)}{\left(\widetilde{\rho}_{k}^{2}-\rho_{n}^{2}\right) J(k ; \nu)}=0, \quad n \in \mathbb{N} .
$$

Further, let

$$
\beta_{k}=\frac{\widetilde{\rho}_{k}^{2}-\rho_{1}^{2}}{\widetilde{\rho}_{k}^{2}}, \quad c_{k}=a_{k} \frac{\widetilde{\rho}_{k}^{\nu+1 / 2} G\left(\widetilde{\rho}_{k}\right) \widetilde{\rho}_{k}^{\nu-1 / 2} P_{\nu}^{\prime}\left(\widetilde{\rho}_{k}\right)}{\widetilde{\rho}_{k}^{2} J^{2}(k ; \nu)}
$$

and

$$
d_{k}=\frac{c_{k}}{\beta_{k}}, \quad b_{k}=a_{k} \frac{2 \widetilde{\rho}_{k}^{\nu+1 / 2} G\left(\widetilde{\rho}_{k}\right)}{\left(\widetilde{\rho}_{k}^{2}-\rho_{1}^{2}\right) J(k ; \nu)} .
$$

Furthermore,

$$
\begin{gathered}
\widetilde{P}(z)=\sum_{k \in \mathbb{N}} \frac{a_{k} \widetilde{\rho}_{k}^{\nu+1 / 2} G\left(\widetilde{\rho}_{k}\right) \widetilde{\rho}_{k}^{\nu-1 / 2} P_{\nu}^{\prime}\left(\widetilde{\rho}_{k}\right)}{J^{2}(k ; \nu)} \frac{2 J(k ; \nu) P_{\nu}(z)}{\widetilde{\rho}_{k}^{\nu-1 / 2} P_{\nu}^{\prime}\left(\widetilde{\rho}_{k}\right)\left(\widetilde{\rho}_{k}^{2}-z^{2}\right)}= \\
=\frac{1}{z^{2}} \sum_{k \in \mathbb{N}} c_{k} \frac{2 J(k ; \nu) P_{\nu}(z)}{\widetilde{\rho}_{k}^{\nu-1 / 2} P_{\nu}^{\prime}\left(\widetilde{\rho}_{k}\right)} \frac{\widetilde{\rho}_{k}^{2} z^{2}}{\widetilde{\rho}_{k}^{2}-z^{2}}= \\
=\frac{1}{z^{2}} \sum_{k \in \mathbb{N}} c_{k} \frac{2 J(k ; \nu) P_{\nu}(z)}{\widetilde{\rho}_{k}^{\nu-1 / 2} \beta_{k} P_{\nu}^{\prime}\left(\widetilde{\rho}_{k}\right)}\left(\frac{\beta_{k} \widetilde{\rho}_{k}^{2} z^{2}}{\widetilde{\rho}_{k}^{2}-z^{2}}-z^{2}\right)+\sum_{k \in \mathbb{N}} c_{k} \frac{2 J(k ; \nu) P_{\nu}(z)}{\widetilde{\rho}_{k}^{\nu-1 / 2} \beta_{k} P_{\nu}^{\prime}\left(\widetilde{\rho}_{k}\right)}= \\
=\left(z^{2}-\rho_{1}^{2}\right) \sum_{k \in \mathbb{N}} d_{k} \frac{2 J(k ; \nu)}{\widetilde{\rho}_{k}^{\nu-1 / 2} P_{\nu}^{\prime}\left(\widetilde{\rho}_{k}\right)} \frac{P_{\nu}(z)}{\widetilde{\rho}_{k}^{2}-z^{2}}+P_{\nu}(z) \sum_{k \in \mathbb{N}} b_{k} .
\end{gathered}
$$


Since (see $[7,8,13])$

$$
\left(z^{-\nu} J_{\nu}(z)\right)^{\prime}=-z^{-\nu} J_{\nu+1}(z), \quad\left|J_{\nu+1}\left(\widetilde{\rho}_{k}\right)\right|=O\left(\widetilde{\rho}_{k}^{-1 / 2}\right)
$$

and, by Lemma $5,\left|\widetilde{\rho}_{k}^{\nu+1 / 2} G\left(\widetilde{\rho}_{k}\right)\right| \leq c_{2}\left(1+\widetilde{\rho}_{k}^{2}\right)$, then

$$
\begin{gathered}
P_{\nu}^{\prime}\left(\widetilde{\rho}_{k}\right)=-\widetilde{\rho}_{k}^{-\nu} J_{\nu+1}\left(\widetilde{\rho}_{k}\right), \\
\left|\widetilde{\rho}_{k}^{\nu-1 / 2} P_{\nu}^{\prime}\left(\widetilde{\rho}_{k}\right)\right| \leq c_{5} \widetilde{\rho}_{k}^{-1}, \quad\left|\frac{\widetilde{\rho}_{k}^{\nu+1 / 2} G\left(\widetilde{\rho}_{k}\right) \widetilde{\rho}_{k}^{\nu-1 / 2} P_{\nu}^{\prime}\left(\widetilde{\rho}_{k}\right)}{J^{2}(k ; \nu)}\right| \leq c_{6} \widetilde{\rho}_{k},
\end{gathered}
$$

and

$$
\sum_{k \in \mathbb{N}}\left|c_{k}\right|^{2}<+\infty, \quad \sum_{k \in \mathbb{N}}\left|d_{k}\right|^{2}<+\infty, \quad \sum_{k \in \mathbb{N}}\left|b_{k}\right|<+\infty .
$$

Moreover, since the system $\left\{J(k ; \nu) \gamma_{k}\left(t ; P_{\nu}\right): k \in \mathbb{N}\right\}$ forms an orthonormal basis in $L^{2}(0 ; 1)$ (see Remark 1) and by Lemmas 1 and 2

$$
\begin{gathered}
\int_{0}^{+\infty} \frac{z^{\nu+1 / 2} 2 J(n ; \nu) P_{\nu}(z)}{\widetilde{\rho}_{n}^{\nu-1 / 2} P_{\nu}^{\prime}\left(\widetilde{\rho}_{n}\right)\left(z^{2}-\widetilde{\rho}_{n}^{2}\right)} \frac{\overline{z^{\nu+1 / 2} 2 J(k ; \nu) P_{\nu}(z)}}{\widetilde{\rho}_{k}^{\nu-1 / 2} P_{\nu}^{\prime}\left(\widetilde{\rho}_{k}\right)\left(z^{2}-\widetilde{\rho}_{k}^{2}\right)} d z= \\
=\int_{0}^{1} J(n ; \nu) \overline{\gamma_{n}\left(t ; P_{\nu}\right)} J(k ; \nu) \gamma_{k}\left(t ; P_{\nu}\right) d t=\delta_{k n},
\end{gathered}
$$

the system

$$
\left\{\frac{2 J(k ; \nu) P_{\nu}(z)}{\widetilde{\rho}_{k}^{\nu-1 / 2} P_{\nu}^{\prime}\left(\widetilde{\rho}_{k}\right)\left(z^{2}-\widetilde{\rho}_{k}^{2}\right)}: k \in \mathbb{N}\right\}
$$

is an orthonormal basis of the space $P W^{2, \nu}$. Therefore, the function

$$
g_{1}(z)=\sum_{k \in \mathbb{N}} d_{k} \frac{2 J(k ; \nu)}{\widetilde{\rho}_{k}^{\nu-1 / 2} P_{\nu}^{\prime}\left(\widetilde{\rho}_{k}\right)} \frac{P_{\nu}(z)}{\widetilde{\rho}_{k}^{2}-z^{2}}
$$

belongs to $P W^{2, \nu}$. The function

$$
g_{2}(z)=\frac{P_{\nu}(z)}{z^{2}-\rho_{1}^{2}} \sum_{k \in \mathbb{N}} b_{k}
$$

also belongs to the space $P W^{2, \nu}$. Hence, the function $\left(z^{2}-\rho_{1}^{2}\right)^{-1} \widetilde{P}(z)$ belongs to $P W^{2, \nu}$ and, taking into account $(2)$, the sequence $\left(\rho_{k}\right)$ is a subsequence of zeros of the function $\widetilde{P}$. This, according to Lemma 3, contradicts to the completeness of the system (1). Theorem 1 is proved.

Remark 2. Let $f \in L^{2}(0 ; 1)$, the system (1) is exact in $L^{2}(0 ; 1)$ and

$$
a_{k}(f):=\int_{0}^{1} \sqrt{t \rho_{k}} J_{\nu}\left(t \rho_{k}\right) \overline{\gamma_{k}(t)} d t, \quad k \in \mathbb{N} .
$$

From Theorem 1 it follows that $f \equiv 0$ if all $a_{k}(f)=0$. Therefore, the function $f \in L^{2}(0 ; 1)$ can be restored by the numbers $a_{k}(f)$. This is very important for finding methods for the reconstruction of $f$ by $a_{k}(f)$ and, in particular, the summation methods of the series

$$
f(x) \sim \sum_{k \in \mathbb{N}} a_{k}(f) \sqrt{x \rho_{k}} J_{\nu}\left(x \rho_{k}\right) .
$$




\section{REFERENCES}

1. N.I. Akhiezer, To the theory of paired integral equations, Uchenye Zapiski Kharkov. Gos. Univ., 25 (1957), 5-31 (in Russian).

2. R.P. Boas, H. Pollard, Complete sets of Bessel and Legendre functions, Ann. of Math., 48 (1947), №2, 366-384.

3. M.M. Dzhrbashyan, Integral transforms and representations of functions in the complex domain, Nauka, Moscow, 1966 (in Russian).

4. J.L. Griffith, Hankel transforms of functions zero outside a finite interval, J. Proc. Roy. Soc. New South Wales, 89 (1955), 109-115.

5. H. Hochstadt, The mean convergence of Fourier-Bessel series, SIAM Rev., 9 (1967), 211-218.

6. B.Ya. Levin, Lectures on entire functions, Transl. Math. Monographs, 150, Amer. Math. Soc., Providence, R.I., 1996.

7. K. Stempak, On convergence and divergence of Fourier-Bessel series, Electron. Trans. Numer. Anal., 14 (2002), 223-235.

8. V.S. Vladimirov, Equations of mathematical physics, Nauka, Moscow, 1981 (in Russian). Engl. transl.: Mir Publishers, Moscow, 1984.

9. B.V. Vynnyts'kyi, R.V. Khats', Completeness and minimality of systems of Bessel functions, Ufa Math. J., 5 (2013), №2, 131-141.

10. B.V. Vynnyts'kyi, R.V. Khats', A remark on basis property of systems of Bessel and Mittag-Leffler type functions, Izv. NAN Armenii, Matematika, 50 (2015), №6, 16-25 (in Russian). Engl. transl.: J. Contemp. Math. Anal., 50 (2015), №6, 300-305.

11. B.V. Vynnyts'kyi, R.V. Khats', On the completeness and minimality of sets of Bessel functions in weighted $L^{2}$-spaces, Eurasian Math. J., 6 (2015), №1, 123-131.

12. B.V. Vynnyts'kyi, R.V. Khats', Some approximation properties of the systems of Bessel functions of index -3/2, Mat. Stud., 34 (2010), №2, 152-159.

13. G.N. Watson, A treatise on the theory of Bessel functions, Cambridge University Press, Cambridge, 1944.

14. R.M. Young, An introduction to nonharmonic Fourier series, Pure and Appl. Math., 93, Academic Press, New York, 1980.

15. R.M. Young, On complete biorthogonal systems, Proc. Amer. Math. Soc., 83 (1981), №3, 537-540.

Institute of Physics, Mathematics, Economy and Innovation Technologies

Drohobych Ivan Franko State Pedagogical University

vynnytskyi@ukr.net

khats@ukr.net 